\section{Stability analysis of solutions to equilibrium problems and applications in economics}

Solutions to equilibrium problems

\author{
Tran Ngoc Tam
}

Can Tho University, Can Tho, Vietnam

Nguyen Minh Hai Banking University of Ho Chi Minh City, Ho Chi Minh, Vietnam, and

Bantaojai Thanatporn

Valaya Alongkorn Rajabhat University under the Royal Patronage, Pathum Thani, Thailand

\begin{abstract}
Purpose - The purpose of this paper is to study the Hölder calmness of solutions to equilibrium problems and apply it to economics.

Design/methodology/approach - The authors obtain the Hölder calmness by using an effective approach. More precisely, under the key assumption of strong convexity, sufficient conditions for the Hölder continuity of solution maps to equilibrium problems are established.

Findings - A new result in stability analysis for equilibrium problems and applications in economics is archived. Originality/value - The authors confirm that the paper has not been published previously, is not under consideration for publication elsewhere and is not being simultaneously submitted elsewhere.
\end{abstract}

Keywords Calmness stability, Equilibrium problem, H5C, Mean-variance portfolio, Nash equilibria, Strong convexity

Paper type Research paper

\section{Introduction}

Many important problems such as optimization problems, variational inequality problems, complementarity problems, Nash equilibrium problems, minimax problems, fixed-point and coincidence-point problems and traffic network problems are considered as special cases of an equilibrium problem (Blum and Oettli, 1994). This implies possibility of a wide application of results in the equilibrium problem theory to several important fields,

(C) Tran Ngoc Tam, Nguyen Minh Hai and Bantaojai Thanatporn. Published in Asian Journal of Economics and Banking. Published by Emerald Publishing Limited. This article is published under the Creative Commons Attribution (CC BY 4.0) licence. Anyone may reproduce, distribute, translate and create derivative works of this article (for both commercial and non-commercial purposes), subject to full attribution to the original publication and authors. The full terms of this licence may be seen at http://creativecommons.org/licences/by/4.0/legalcode

JEL classification - C62, B23, D58

The authors are very grateful to the referee for his valuable remarks and suggestions, which helped us improve the paper.
Received 27 June 2020 Revised 8 August 2020 Accepted 8 August 2020 
AJEB

4,3

including economics, physics (especially, mechanics), engineering, transportation, sociology, chemistry, biology, etc. (Kassay and Radulescu, 2018).

Existence conditions of solutions for the equilibrium problem and its generalizations are the first and most developed topic. There are a lot of works devoted to this topic in the literature, see e.g. Ansari et al. (2001), Castellani et al. (2010), Hai and Khanh (2007), Jafari et al. (2017), Hai et al. (2009), Sadeqi and Alizadeh (2011) and Alleche and Rădulescu (2016), and the references therein. The second one is stability analysis of solutions. Stability conditions, especially the lower semicontinuity and Hölder continuity of solution maps, for such problems have been extensively studied and received increasing attention from many researchers so far (Bianchi and Rita, 2006; Anh and Khanh, 2008; Anh and Khanh, 2010; Kimura and Yao, 2008; Kimura and Yao, 2008; Li et al., 2013; Anh et al., 2018; and references therein).

The paper aims at investigating the stability analysis in the sense of Hölder calmness of the solution maps to equilibrium problems. To be more precise, sufficient conditions for the solution maps to be Hölder calm are established. At the end of the paper, we present applications of the main results in economics. Namely, the Hölder calmness of solution maps to mean-variance portfolio and Nash equilibrium problems is derived.

The rest of the paper is organized as follows. Section 2 states the equilibrium problem setting and recalls some definitions and their properties needed in what follows. Sufficient conditions for the Hölder calmness of the solution maps are established in Section 3. In Section 4, we discuss the Hölder calmness of solution maps to mean-variance portfolio and Nash equilibrium problems.

\section{Preliminaries}

In this paper, we use $d(\cdot, \cdot)$ for the metric in metric spaces. For two subsets $A, B \subseteq X$, we use the following notations:

$$
\begin{gathered}
d(a, B):=\inf _{b \in B} d(a, b), \\
H^{*}(A, B):=\sup _{a \in A} d(a, B), \\
H(A, B):=\max \left\{H^{*}(A, B), H^{*}(B, A)\right\}, \\
\rho(A, B):=\sup _{a \in A, b \in B} d(a, b) .
\end{gathered}
$$

Denote $\mathbb{R}_{+}$is the set of the nonnegative real numbers and $\mathbb{B}(x, r)$ is the closed ball of radius $r \geq 0$ and is centered at $x . \operatorname{int} A, \operatorname{conv}(A)$ and $\operatorname{diam} A:=\sup _{x, z \in A} d(x, z)$ stand for the interior, the convex hull and the diameter, respectively, of a subset $A$. For a set-valued map $G: X \rightrightarrows Y$, $g p h G:=\{(x, y) \in X \times Y: y \in G(x)\}$ is the graph of $G$. Recall that $X$ is called a metric linear space if and only if it is both a metric space and a linear space and the metric $d$ of $X$ is translation invariant (i.e. $d(x+z, y+z)=d(x, y), \forall x, y, z \in X)$ and, for any convergent sequences $\left(\lambda_{m}\right)$ in $\mathbb{R}$ and $\left(x^{m}\right)$ in $X$, we have $\lim _{m}\left(\lambda_{m} x^{m}\right)=\left(\lim _{m} \lambda_{m}\right)\left(\lim _{m} x^{m}\right)$. 
From now on, unless otherwise stated, let $X$ be a metric linear space, $\Lambda, M$ be metric spaces and $A \subseteq X$ be nonempty. Let $K: \Lambda \rightrightarrows A$ be nonempty-convex-valued and $\varphi: A \times A \times M \rightarrow \mathbb{R}$. For $(\lambda, \mu) \in \Lambda \times M$, we consider the following parametric equilibrium problem, i.e. the family of the corresponding problems when $(\lambda, \mu)$ varies in $\Lambda \times M$,

(EP) find $\bar{x} \in K(\lambda)$ such that $\varphi(\bar{x}, y, \mu) \geq 0$ for all $y \in K(\lambda)$.

For each $(\lambda, \mu) \in \Lambda \times M$, denote the solution set of (EP) by $S(\lambda, \mu)$, i.e.:

$$
S(\lambda, \mu):=\{x \in K(\lambda): \varphi(x, y, \mu) \geq 0, \forall y \in K(\lambda)\} .
$$

As existence of solutions has been studied much in the literature, we do not include existence investigations and always assume that $S(\lambda, \mu)$ is nonempty in the neighborhood of the considered point.

We first recall some notions needed in the sequel.

Definition 2.1. Let $n, \gamma>0$ and $\theta \geq 0$. It is said that:

- a function $g: X \rightarrow \mathbb{R}$ is $n . \gamma$-Hölder continuous at $\bar{x} \in X$ if there is a neighborhood $U$ of $\bar{x}$ such that, for all $x_{1}, x_{2} \in U$ :

$$
d\left(g\left(x_{1}\right), g\left(x_{2}\right)\right) \leq n d^{\gamma}\left(x_{1}, x_{2}\right)
$$

- a function $g: X \rightarrow \mathbb{R}$ is $n . \gamma$-Hölder calm at $\bar{x} \in X$ if there is a neighborhood $U$ of $\bar{x}$ such that, for all $x \in U$ :

$$
d(g(x), g(\bar{x})) \leq n d^{\gamma}(x, \bar{x})
$$

- a function $g: X \times X \times M \rightarrow \mathbb{R}$ is $n . \gamma$-Hölder calm at $\bar{\mu} \in M, \theta$-uniformly over a subset $B \subseteq X$ if there is a neighborhood $U$ of $\bar{\mu}$ such that, for all $\mu \in U$ and $x, y \in B: x \neq y$ :

$$
d(g(x, y, \mu), g(x, y, \bar{\mu})) \leq n d^{\gamma}(\mu, \bar{\mu}) d^{\theta}(x, y)
$$

if $\theta=0$, we say that $g(x, y, \cdot)$ is $n$. $\gamma$-Hölder calm at $\bar{\mu}$, uniformly over $B$; and

- a multifunction $K: \Lambda \rightrightarrows X$ is $n . \gamma$-Hölder calm at $\bar{\lambda} \in \Lambda$ if there exists a neighborhood $N$ of $\bar{\lambda}$ such that, for all $\lambda \in N$ :

$$
H(K(\lambda), K(\bar{\lambda})) \leq d^{\gamma}(\lambda, \bar{\lambda})
$$

If $\gamma=1$, then Hölder continuity is called Lipschitz continuity.

We say that a certain property is satisfied in a subset $B \subseteq X$ if and only if it is satisfied at every point of $B$.

Definition 2.2. Let $g: X \rightarrow \mathbb{R}, B \subseteq X$, and $h, \beta$ be positive.

- $g$ is called $h \cdot \beta$-strongly convex on a convex subset $B$ if and only if, for all $x_{1}, x_{2} \in B$ and $t \in(0,1)$ :

$$
g\left((1-t) x_{1}+t x_{2}\right) \leq(1-t) g\left(x_{1}\right)+\operatorname{tg}\left(x_{2}\right)-h t(1-t) d^{\beta}\left(x_{1}, x_{2}\right) .
$$


AJEB

4,3

- $g$ is called $h . \beta$-strongly convex-like in $B$ ( $B$ not necessarily convex) if and only if, for all $x_{1}, x_{2} \in B$ and $t \in(0,1)$, there is $z \in B$ such that:

$$
g(z) \leq(1-t) g\left(x_{1}\right)+\operatorname{tg}\left(x_{2}\right)-h t(1-t) d^{\beta}\left(x_{1}, x_{2}\right) .
$$

Recall that a function $g: X \times X \rightarrow \mathbb{R}$ is called monotone on $B \subseteq X$ if:

$$
g(x, y)+g(y, x) \leq 0, \forall x, y \in B .
$$

In what follows, we use the following assumptions that play an important role in investigating conditions for the Hölder calmness of solution maps to the equilibrium problems:

H1. $K$ is $l . \alpha$-Hölder calm on $\Lambda$.

H2. The map $y \mapsto \varphi(x, y, \mu)$ is $h$. $\beta$-strongly convex as well as $m$.1-Hölder continuous in $\operatorname{conv}(K(\Lambda))$.

H3. The map $(x, y) \mapsto \varphi(x, y, \mu)$ is monotone on $K(\Lambda) \times K(\Lambda)$ for all $\mu \in M$.

H4. The map $\mu \mapsto \varphi(x, y, \mu)$ is $n$. $\gamma$-Hölder calm on $M, \theta$-uniformly over $K(\Lambda)$.

\section{Hölder calmness of solutions}

In this section, we state the main results of the paper. Namely, we establish sufficient conditions for the Hölder calmness of the solution maps to equilibrium problems.

Theorem 3.1. Assume that all assumptions $(H 1-H 4)$ are satisfied with $\theta<\beta$. Then, the solution map $\mathrm{S}$ is single-valued and Hölder calm on $\Lambda \times \mathrm{M}$.

Proof.

Let $(\overline{\bar{\lambda}}, \bar{\mu}) \in \Lambda \times M$ be arbitrary, we need to prove that $S$ is Hölder calm at $(\bar{\lambda}, \bar{\mu})$. We divide the proof into three steps.

Step 1. For all $x_{11} \in S(\bar{\lambda}, \bar{\mu})$ and $x_{21} \in S(\lambda, \bar{\mu})$, we claim that:

$$
d_{1}:=d\left(x_{11}, x_{21}\right) \leq\left(\frac{4 m l}{h}\right)^{\frac{1}{\beta}} d^{\frac{\alpha}{\beta}}(\bar{\lambda}, \lambda) .
$$

By the definition of the solution set, we have, for all $y \in K(\bar{\lambda})$ and $z \in K(\lambda)$ :

$$
\min \left\{\varphi\left(x_{11}, y, \bar{\mu}\right), \varphi\left(x_{21}, z, \bar{\mu}\right)\right\} \geq 0 .
$$

The Hölder calmness of $K$ leads to the existence of $x_{1} \in K(\bar{\lambda})$ and $x_{2} \in K(\lambda)$ such that:

$$
\max \left\{d\left(x_{11}, x_{2}\right), d\left(x_{21}, x_{1}\right)\right\} \leq l d^{\alpha}(\bar{\lambda}, \lambda) .
$$

Letting $\hat{x}=\frac{1}{2}\left(x_{11}+x_{21}\right)$, it follows from the strong convexity in $H 2$ that:

$$
\varphi\left(x_{11}, \hat{x}, \bar{\mu}\right) \leq \frac{1}{2} \varphi\left(x_{11}, x_{11}, \bar{\mu}\right)+\frac{1}{2} \varphi\left(x_{11}, x_{21}, \bar{\mu}\right)-\frac{1}{4} h d_{1}^{\beta} .
$$

By virtue of the monotonicity of $\varphi$ and $x_{11} \in S(\bar{\lambda}, \bar{\mu})$, one gets:

$$
f\left(x_{11}, x_{11}, \bar{\mu}\right)=0
$$




$$
f\left(x_{11}, x_{21}, \bar{\mu}\right) \leq-f\left(x_{21}, x_{11}, \bar{\mu}\right) .
$$

Hence, equation (4) implies that:

$$
\frac{1}{4} h d_{1}^{\beta} \leq-\frac{1}{2} \varphi\left(x_{21}, x_{11}, \bar{\mu}\right)-\varphi\left(x_{11}, \frac{x_{11}+x_{21}}{2}, \bar{\mu}\right) .
$$

Letting $z=x_{2}$ and $y=\frac{1}{2}\left(x_{11}+x_{1}\right)$ in equation (2), one has:

$$
\min \left\{\frac{1}{2} \varphi\left(x_{21}, x_{2}, \bar{\mu}\right), \varphi\left(x_{11}, \frac{x_{11}+x_{1}}{2}, \bar{\mu}\right)\right\} \geq 0
$$

From equations (5) and (6), we arrive at:

$$
\begin{aligned}
\frac{1}{4} h d_{1}^{\beta} & \leq \frac{1}{2}\left[\varphi\left(x_{21}, x_{2}, \bar{\mu}\right)-\varphi\left(x_{21}, x_{11}, \bar{\mu}\right)\right] \\
+ & {\left[\varphi\left(x_{11}, \frac{x_{11}+x_{1}}{2}, \bar{\mu}\right)-\varphi\left(x_{11}, \frac{x_{11}+x_{21}}{2}, \bar{\mu}\right)\right] . }
\end{aligned}
$$

Hence, using the $m$.1-Hölder calmness in H2 and equation (3), we obtain:

$$
\begin{aligned}
\frac{1}{4} h d_{1}^{\beta} \leq & \frac{1}{2} m d\left(x_{11}, x_{2}\right)+\frac{1}{2} m d\left(x_{21}, x_{1}\right) \\
& \leq \frac{1}{2} m l d^{\alpha}(\bar{\lambda}, \lambda)+\frac{1}{2} m l d^{\alpha}(\bar{\lambda}, \lambda)
\end{aligned}
$$

that is:

$$
\frac{1}{4} h d_{1}^{\beta} \leq m l d^{\alpha}(\bar{\lambda}, \lambda)
$$

Hence, we obtain (1).

Step 2. We will show that, for all $x_{21} \in S(\lambda, \bar{\mu})$ and $x_{22} \in S(\lambda, \mu)$ :

$$
d_{2}:=d\left(x_{21}, x_{22}\right) \leq\left(\frac{n}{h}\right)^{\frac{1}{\beta-\theta}} d^{\frac{\gamma}{\beta-\theta}}(\bar{\mu}, \mu) .
$$

By the definition of the solution, one has, for all $y, z \in K(\lambda)$,

$$
\min \left\{\varphi\left(x_{21}, y, \bar{\mu}\right), \varphi\left(x_{22}, z, \mu\right)\right\} \geq 0 .
$$

Putting $y=\frac{1}{2}\left(x_{22}+x_{21}\right)$ in equation (8), we have:

$$
\varphi\left(x_{21}, \frac{x_{22}+x_{21}}{2}, \bar{\mu}\right) \geq 0 .
$$

By the strong convexity given in $H 2$, we have: 


$$
f\left(x_{21}, x_{22}, \bar{\mu}\right)+f\left(x_{21}, x_{21}, \bar{\mu}\right)-\frac{1}{2} h d_{2}^{\beta} \geq 0 .
$$

The monotonicity of $\varphi$ assumed in $H 3$ and $x_{21} \in S(\lambda, \bar{\mu})$ imply that:

$$
\varphi\left(x_{21}, x_{21}, \bar{\mu}\right)=0
$$

$$
\varphi\left(x_{21}, x_{22}, \bar{\mu}\right) \leq-\varphi\left(x_{22}, x_{21}, \bar{\mu}\right) .
$$

Hence, combining with equation (9), we have:

$$
\frac{1}{2} h d_{2}^{\beta} \leq-\varphi\left(x_{22}, x_{21}, \bar{\mu}\right)
$$

Now, letting $z=\frac{1}{2}\left(x_{22}+x_{21}\right)$ in equation (8) derives:

$$
\varphi\left(x_{22}, \frac{x_{22}+x_{21}}{2}, \mu\right) \geq 0 .
$$

Also by the strong convexity given in $H 2$, we have:

$$
\varphi\left(x_{22}, x_{22}, \mu\right)+\varphi\left(x_{22}, x_{21}, \mu\right)-\frac{1}{2} h d_{2}^{\beta} \geq 0 .
$$

As $\varphi\left(x_{22}, x_{22}, \mu\right)=0$, this yields that:

$$
\frac{1}{2} h d_{2}^{\beta} \leq \varphi\left(x_{22}, x_{21}, \mu\right)
$$

Adding equation (10) and this inequality, and using assumption $H 4$, we get:

$$
\begin{aligned}
h d_{2}^{\beta} & \leq f\left(x_{22}, x_{21}, \mu\right)-f\left(x_{22}, x_{21}, \bar{\mu}\right) \\
& \leq \quad n d^{\gamma}(\bar{\mu}, \mu) d_{2}^{\theta},
\end{aligned}
$$

i.e. we obtain equation (7).

Step 3. We are now ready to complete the proof. For all $x_{11} \in S(\bar{\lambda}, \bar{\mu})$ and $x_{22} \in S(\lambda, \mu)$, we have:

$$
d\left(x_{11}, x_{22}\right) \leq d_{1}+d_{2}
$$

Hence, from equations (1) and (7), we get, with $k_{1}=\left(\frac{4 m l}{h}\right)^{\frac{1}{\beta}}$ and $k_{2}=\left(\frac{n}{h}\right)^{\frac{1}{\beta-\theta}}$ :

$$
\rho(S(\bar{\lambda}, \bar{\mu}), S(\lambda, \mu)) \leq k_{1} d^{\frac{\alpha}{\beta}}(\bar{\lambda}, \lambda)+k_{2} d^{\frac{\gamma}{\beta-\theta}}(\bar{\mu}, \mu) .
$$

Letting $\bar{\lambda}=\lambda$ and $\bar{\mu}=\mu$ in this inequality, we see that the diameter of $S(\bar{\lambda}, \bar{\mu})$ is 0 [for arbitrary $(\bar{\lambda}, \bar{\mu})$ ], i.e. the solution map of (EP) is single-valued in $\Lambda \times M$. The proof is complete. 
In the special case where $K(\lambda) \equiv K$ ( $K$ is a nonempty set), we have the following result.

Theorem 3.2. For (EP) with $K(\lambda) \equiv K$, assume that assumptions $H 3$ and $H 4$ are satisfied and $H 2$ is replaced by the following condition:

$H^{\prime} 2$ : The map $y \mapsto \varphi(x, y, \mu)$ is $h$. $\beta$-strongly convex-like on $K$.

Then, $S$ is Hölder calm on $\Lambda \times M$.

Proof. For any $x_{1} \in S(\bar{\mu}), x_{2} \in S(\mu)$ and $y, z \in K$, one has:

$$
\min \left\{\varphi\left(x_{1}, y, \bar{\mu}\right), \varphi\left(x_{2}, z, \mu\right)\right\} \geq 0 .
$$

By the strong convex-likeness of $f$ in $K$, there is $\bar{z} \in K$ such that:

$$
\varphi\left(x_{1}, \bar{z}, \bar{\mu}\right) \leq(1-t) \varphi\left(x_{1}, x_{2}, \bar{\mu}\right)+t \varphi\left(x_{1}, x_{1}, \bar{\mu}\right)-h t(1-t) d^{\beta}\left(x_{1}, x_{2}\right) .
$$

This and the monotonicity of $\varphi$ imply that:

$$
h t(1-t) d^{\beta}\left(x_{1}, x_{2}\right) \leq-(1-t) \varphi\left(x_{2}, x_{1}, \bar{\mu}\right)-\varphi\left(x_{1}, \bar{z}, \bar{\mu}\right) .
$$

As $\varphi\left(x_{1}, \bar{z}, \bar{\mu}\right) \geq 0$, this inequality leads to:

$$
h t d^{\beta}\left(x_{1}, x_{2}\right) \leq-\varphi\left(x_{2}, x_{1}, \bar{\mu}\right) .
$$

With the same arguments but with $x_{1}$ replaced by $x_{2}$ and $\bar{\mu}$ by $\mu$ in equation (11), we have:

$$
h(1-t) d^{\beta}\left(x_{1}, x_{2}\right) \leq \varphi\left(x_{2}, x_{1}, \mu\right) .
$$

Adding equations (12) and (13), and using assumption $H 3$, one gets:

$$
\begin{aligned}
h d^{\beta}\left(x_{1}, x_{2}\right) & \leq \varphi\left(x_{2}, x_{1}, \mu\right)-\varphi\left(x_{2}, x_{1}, \bar{\mu}\right) \\
& \leq n d^{\gamma}(\bar{\mu}, \mu) d^{\theta}\left(x_{1}, x_{2}\right) .
\end{aligned}
$$

This means the required Hölder condition. From this, we obtain the uniqueness of the solution.

\section{Applications}

\subsection{Mean-variance portfolio}

In this subsection, we present an application of our results to a practical situation. Namely, we study Hölder properties of solution maps to parametric quadratic programing, a special case of the equilibrium problem, which has well-known applications in the view of practice. A good sample is the model of mean-variance portfolio based on investor's utility maximization. This problem can be formulated as parametric quadratic optimization problems. Consider a universe of $n$ assets with the known data is $c=\left(c_{1}, \ldots, c_{n}\right)^{T}$ and $Q=\left[\sigma_{i j}\right]$, where $c_{i}$ is the expected return for asset $i$ and $\sigma_{i j}$ is the covariance of returns for assets $i$ and $j$. So, $c$ is the vector of expected returns and $Q$ is the $n \times n$ variance-covariance matrix of asset returns. Herein, $Q$ is a positive definite matrix which follows from the properties of variance-covariance matrices. Denote the vector of asset holdings by $x=\left(x_{1}, \ldots, x_{n}\right)^{T}$. Then, the expected return of the portfolio $x$ is $c^{T x}$ and its variance is $\sigma^{2}=x^{T} Q x$. A portfolio is said to be efficient if for some fixed level of expected return no 
AJEB

4,3

other portfolio gives smaller variance (Markowitz, 1956). In other words, an efficient portfolio can be considered as the one for which at some fixed level of variance no other portfolio gives larger expected return. The determination of the efficient portfolio frontier in this mean-variance model is equivalent to solving the following parametric problem because of (Farrar, 1965):

$$
(\mathrm{MP}) \quad \min f(x, \mu):=\frac{1}{2} x^{T} Q x-\mu c^{T} x \quad \text { s.t. } \quad \mathrm{Ax}=\mathrm{b}, \mathrm{x} \in \mathbb{R}_{+}^{\mathrm{n}},
$$

where $\mu \geq 0$ is an investor's variance aversion parameter. The linear constraints $A x=b$ can represent budget constraints, bounds on asset holdings, etc. Nonnegativity constraints $x \geq 0$ are short-sale constraints (non-negative asset holdings).

Lemma 4.1. If $u(x)$ is $h$. $\beta$-strongly convex and $v(x)$ is convex on a convex set $A \subset \mathbb{R}^{n}$, then $g(x)=u(x)+v(x)$ is $h \cdot \beta$-strongly convex on $\mathrm{A}$.

Proof. For all $x_{1}, x_{2} \in A$ and $t \in[0,1]$, we have:

$$
\begin{gathered}
g\left(t x_{1}+(1-t) x_{2}\right)=u\left(t x_{1}+(1-t) x_{2}\right)+v\left(t x_{1}+(1-t) x_{2}\right) \\
\leq t u\left(x_{1}\right)+(1-t) u\left(x_{2}\right)-h t(1-t) d^{\beta}\left(x_{1}, x_{2}\right)+t v\left(x_{1}\right) \\
+(1-t) v\left(x_{2}\right) \\
\leq t\left(u\left(x_{1}\right)+v\left(x_{1}\right)\right)+(1-t)\left(u\left(x_{2}\right)+v\left(x_{2}\right)\right) \\
-h t(1-t) d^{\beta}\left(x_{1}, x_{2}\right) \\
\leq \operatorname{tg}\left(x_{1}\right)+(1-t) g\left(x_{2}\right)-h t(1-t) d^{\beta}\left(x_{1}, x_{2}\right) .
\end{gathered}
$$

Hence, $g$ is a strongly convex function.

Lemma 4.2. The function $g(x)=x^{T} Q x$ is strongly convex with respect to the Euclidean norm in $\mathbb{R}^{n}$, where $Q$ is a positive definite matrix.

Proof. As $Q$ is a positive definite matrix, we can consider the norm defined by $\|x\|=\sqrt{x^{T} Q x}$ on $\mathbb{R}^{n}$. We first prove that the function $g(x)=x^{T} Q x$ is strongly convex with respect to this norm. Indeed, for all $x_{1}, x_{2} \in \mathbb{R}^{n}$ and $t \in[0,1]$, one has:

$$
\begin{gathered}
g\left(t x_{1}+(1-t) x_{2}\right)=\left(t x_{1}+(1-t) x_{2}\right)^{T} Q\left(t x_{1}+(1-t) x_{2}\right) \\
=t^{2} x_{1}^{T} Q x_{1}+t(1-t) x_{1}^{T} Q x_{2}+t(1-t) x_{2}^{T} Q x_{1} \\
+(1-t)^{2} x_{2}^{T} Q x_{2} \\
=t^{2} x_{1}^{T} Q x_{1}+(1-t)^{2} x_{2}^{T} Q x_{2}+2 t(1-t) x_{1}^{T} Q x_{2}
\end{gathered}
$$




$$
\begin{gathered}
=t x_{1}^{T} Q x_{1}+(1-t) x_{2}^{T} Q x_{2} \\
-t(1-t)\left(x_{1}^{T} Q x_{1}+x_{2}^{T} Q x_{2}-2 x_{1}^{T} Q x_{2}\right) \\
=t x_{1}^{T} Q x_{1}+(1-t) x_{2}^{T} Q x_{2}-t(1-t)\left\|x_{1}-x_{2}\right\|^{2} \\
=\operatorname{tg}\left(x_{1}\right)+(1-t) g\left(x_{2}\right)-t(1-t)\left\|x_{1}-x_{2}\right\|^{2} .
\end{gathered}
$$

We now show that $g(x)=x^{T} Q x$ is strongly convex with respect to the Euclidean norm $\|\cdot\|_{E}$. As any two norms on a finite dimensional normed space are equivalent, there are $a$, $b>0$ such that $a\|\cdot\|_{E} \leq\|\cdot\| \leq b\|\cdot\|_{E}$. Therefore, for all $x_{1}, x_{2} \in \mathbb{R}^{n}$ and $t \in[0,1]$, we get:

$$
g\left(t x_{1}+(1-t) x_{2}\right) \leq \operatorname{tg}\left(x_{1}\right)+(1-t) g\left(x_{2}\right)-a^{2} t(1-t)\left\|x_{1}-x_{2}\right\|_{E}^{2} .
$$

The proof is complete.

If we set $\varphi(x, y, \mu):=f(y, \mu)-f(x, \mu)$, then (MP) becomes the parametric equilibrium problem. For $\mu \geq 0$, we denote the solution set of (MP) by $S_{1}(\mu)$.

Lemma 4.3. If the function $y \mapsto g(y)$ is strongly convex, then the function $y \mapsto \varphi(x, y):=f(y)-f(x)$ is also strongly convex.

Proof. We omit the proof as it is trivial.

We have the result for the Hölder calmness of the solution map $S_{1}(\mu)$ as follows:

Corollary 4.1. If $Q$ is a positive definite matrix then the solution map $S_{1}$ is Hölder calm on $(0,+\infty)$.

Proof. We prove this corollary by checking all assumptions of Theorem 3.1. We see that assumptions $H 1, H 3$ and $H 4$ are obviously fulfilled. The strong convexity and Hölder calmness in $H 2$ is derived from Lemmas 4.1, 4.2 and 4.3.

\subsection{Nash equilibrium problem}

In this subsection, we discuss an application of the main results to a Nash equilibrium problem (Debreu, 1952; Facchinei and Kanzow, 2007). Let us state the model of the problem as follows. Let $I \subset \mathbb{N}$ be an index set, and $\Lambda, M, X_{i}(i \in I)$ be linear metric vector spaces. We use the notations $X=\prod_{i \in I} X_{i}$ and $X_{-i}=\prod_{j \in I, j \neq i} X_{j}$. For each $x \in X$, we denote its $i$ th coordinate and its projection on $X_{-i}$ by $x_{i}$ and $x_{-i}$, respectively. We also denote an element of $X$ by $\left(x_{i}, x_{-i}\right)$. For $i \in I$, let $f_{i}$ be extended real-valued functions defined on $X \times M$ and $K_{i}$ be set-valued mappings from $\Lambda$ into $X_{i}$. For any fixed $(\lambda, \mu) \in \Lambda \times M$, an abstract economy, introduced by Debreu (1952), is the set of data:

$$
\Gamma(\lambda, \mu)=\left\{X_{i}, K_{i}(\lambda), f_{i}(\cdot, \mu): i \in I\right\} .
$$

A profile of strategies $x^{*} \in X$ is said to be a social Nash equilibrium of $\Gamma(\lambda, \mu)$ if for any $i \in I, x^{*} \in K(\lambda)$ and:

$$
f_{i}\left(x^{*}, \mu\right)=\max _{y_{i} \in K(\lambda)} f_{i}\left(\left(y_{i}, x_{-i}^{*}\right), \mu\right) .
$$


For each $(\lambda, \mu) \in \Lambda \times M$, the set of all social Nash equilibria of the parametric abstract economy $\Gamma(\lambda, \mu)$ is denoted by $\mathcal{N}(\lambda, \mu)$. The set-valued mapping $\mathcal{N}: \Lambda \times M \rightrightarrows X$ is called a social Nash equilibria set-valued mapping. The following lemma gives us the equivalence between the social Nash equilibrium problem and (EP).

Lemma 4.4. For given $(\bar{\lambda}, \bar{\mu}) \in \Lambda \times M, x^{*}$ is a Nash equilibrium of abstract economy $\Gamma(\bar{\lambda}, \bar{\mu})$ if and only if $x^{*} \in K(\bar{\lambda})$ and:

$$
\varphi\left(x^{*}, y, \bar{\mu}\right) \geq 0, \quad \forall y \in K(\bar{\lambda}),
$$

i.e. $x *$ is a solution to $(\mathrm{EP})$, where:

$$
K(\bar{\lambda}):=\prod_{i \in I} K_{i}(\bar{\lambda}) \text { and } \varphi(x, y, \bar{\mu})=\sum_{i \in I}\left[f_{i}\left(\left(x_{i}, x_{-i}\right), \bar{\mu}\right)-f_{i}\left(\left(y_{i}, x_{-i}\right), \bar{\mu}\right)\right] .
$$

Proof. If equation (14) holds for $\bar{\lambda} \in \Lambda$, then it is obvious that $x^{*}$ is a solution to (EP). Conversely, let $x^{*} \in X$ be such that $x^{*} \in K(\bar{\lambda})$ and equation (15) holds. For each $i \in I$, we choose $\hat{y} \in X$ in such a way satisfying $\hat{y}_{i}=y_{i} \in K_{i}(\bar{\lambda})$ and $\hat{y}_{j}=x_{j}^{*}$ for all $j \in I \backslash\{i\}$. Then, $\hat{y} \in K(\bar{\lambda})$ and:

$$
\begin{gathered}
\varphi\left(x^{*}, \hat{y}, \bar{\mu}\right)=\sum_{i \in I}\left[f_{i}\left(\left(x_{i}^{*}, x_{-i}^{*}\right), \bar{\mu}\right)-f_{i}\left(\left(\hat{y}_{i}, x_{-i}^{*}\right), \bar{\mu}\right)\right] \\
=\sum_{j \in I}^{j \neq i}\left[f_{j}\left(\left(x_{j}^{*}, x_{-j}^{*}\right), \bar{\mu}\right)-f_{j}\left(\left(\hat{y}_{j}, x_{-j}^{*}\right), \bar{\lambda}\right)\right]+f_{i}\left(\left(x_{i}^{*}, x_{-i}^{*}\right), \bar{\mu}\right)-f_{i}\left(\left(\hat{y}_{i}, x_{-i}^{*}\right), \bar{\mu}\right) \\
=f_{i}\left(\left(x_{i}^{*}, x_{-i}^{*}\right), \bar{\mu}\right)-f_{i}\left(\left(\hat{y}_{i}, x_{-i}^{*}\right), \bar{\mu}\right) .
\end{gathered}
$$

Combining this with equation (15), we derive $f_{i}\left(\left(x_{i}^{*}, x_{-i}^{*}\right), \bar{\mu}\right) \geq f_{i}\left(\left(y_{i}, x_{-i}^{*}\right), \bar{\mu}\right)$ for all $y_{i} \in K_{i}(\bar{\lambda})$ and $i \in I$. Consequently, we conclude that for each $i \in I, x^{*} \in K(\bar{\lambda})$ and $f_{i}\left(x^{*}, \bar{\mu}\right)=\max _{y_{i} \in K_{i}(\bar{\lambda})} f_{i}\left(\left(y_{i}, x_{-i}^{*}\right), \bar{\mu}\right)$, i.e. $x^{*}$ is a social Nash equilibrium of abstract economy $\Gamma(\bar{\lambda}, \bar{\mu})$.

Lemma 4.4 allows us to apply Theorem 3.1 to the mapping $\mathcal{N}$, obtaining the following result.

Theorem 4.1. Assume that the set-valued map $K: \Lambda \rightrightarrows X$, and the function $\varphi: X \times X \times \Lambda \rightarrow \mathbb{R} \cup\{\infty\}$ defined by:

$$
K(\lambda):=\prod_{i \in I} K_{i}(\lambda)
$$

and:

$$
\varphi(x, y, \lambda):=\sum_{i \in I}\left[f_{i}\left(\left(x_{i}, x_{-i}\right), \mu\right)-f_{i}\left(\left(y_{i}, x_{-i}\right), \mu\right)\right],
$$

respectively, satisfy conditions (i)-(iv) of Theorem 3.1 on $\Lambda \times M$. Then, the set-valued mapping $\mathcal{N}$ is Hölder calm on $\Lambda \times M$. 


\section{Conclusions}

In this paper, we are successful in establishing the Hölder calmness of solution maps to equilibrium problems where both objective functions and constraints are perturbed. Our results are new. These main results are applied to some special cases including meanvariance portfolio and Nash equilibrium problem. Tools used in this paper can be used for investigating more generalized settings.

\section{References}

Alleche, B. and Rădulescu, V. (2016), "Solutions and approximate solutions of quasi-equilibrium problems in Banach spaces", Journal of Optimization Theory and Applications, Vol. 170 No. 2, pp. 629-649.

Anh, L.Q. and Khanh, P.Q. (2008), "Various kinds of semicontinuity and solution sets of parametric multivalued symmetric vector quasi equilibrium problems", Journal of Global Optimization, Vol. 41 No. 4, pp. 539-558.

Anh, L.Q. and Khanh, P.Q. (2010), "Continuity of solution maps of parametric quasi equilibrium problems", Journal of Global Optimization, Vol. 46 No. 2, pp. 247-259.

Anh, L.Q., Duoc, P.T. and Tam, T.N. (2018), "On Hölder continuity of solution maps to parametric vector primal and dual equilibrium problems", Optimization, Vol. 67 No. 8, pp. 1169-1182.

Ansari, Q.H., Konnov, I.V. and Yao, J.C. (2001), "Existence of a solution and variational principles for vector equilibrium problems", Journal of Optimization Theory and Applications, Vol. 110 No. 3, pp. 481-492.

Bianchi, M. and Rita, P. (2006), "Sensitivity for parametric vector equilibria", Optimization, Vol. 55 No. 3, pp. 221-230.

Blum, E. and Oettli, W. (1994), "From optimization and variational inequalities to equilibrium problems", Math Student, Vol. 63, pp. 123-145.

Castellani, M., Pappalardo, M. and Passacantando, M. (2010), "Existence results for nonconvex equilibrium problems", Optim Meth Software, Vol. 25 No. 1, pp. 49-58.

Debreu, G. (1952), “A social equilibrium existence theorem”, Proceedings of the National Academy of Sciences, Vol. 38 No. 10, pp. 886-893.

Facchinei, F. and Kanzow, C. (2007), "Generalized Nash equilibrium problems”, 4OR, Vol. 5 No. 3, pp. 173-210.

Farrar, D.E. (1965), The Investment Decision under Uncertainty, Prentice-Hall, New York, NY.

Hai, N.X., Khanh, P.Q. and Quan, N.H. (2009), "On the existence of solutions to quasi variational inclusion problems”, Journal of Global Optimization, Vol. 45 No. 4, pp. 565-581.

Hai, N.X. and Khanh, P.Q. (2007), "Existence of solutions to general quasi equilibrium problems and applications", Journal of Optimization Theory and Applications, Vol. 133 No. 3, pp. 317-327.

Jafari, S., Farajzadeh, A.P., Moradi, S. and Khanh, P.Q. (2017), "Existence results for $\varphi$-quasi monotone equilibrium problems in convex metric spaces", Optimization, Vol. 66 No. 3, pp. 293-310.

Kassay, G. and Radulescu, V.D. (2018), Equilibrium Problems and Applications, Academic Press, New York, NY.

Kimura, K. and Yao, J.C. (2008), "Sensitivity analysis of solution mappings of parametric vector quasiequilibrium problems", Journal of Global Optimization, Vol. 41 No. 2, pp. 187-202.

Kimura, K. and Yao, J.C. (2008), "Semicontinuity of solution mappings of parametric generalized vector equilibrium problems", Journal of Optimization Theory and Applications, Vol. 138 No. 3, pp. $429-443$. 
AJEB

4,3

132

Li, S.J., Liu, H.M., Zhang, Y. and Fang, Z.M. (2013), "Continuity of solution mappings to parametric generalized strong vector equilibrium problems", Journal of Global Optimization, Vol. 55 No. 3, pp. 597-610.

Markowitz, H.M. (1956), "The optimization of a quadratic function subject to linear constraints", Naval Research Logistics Quarterly, Vol. 3 Nos 1/2, pp. 111-133.

Sadeqi, I. and Alizadeh, C.G. (2011), "Existence of solutions of generalized vector equilibrium problems in reflexive Banach spaces”, Nonlinear Anal, Vol. 74 No. 6, pp. 2226-2234.

\section{Corresponding author}

Tran Ngoc Tam can be contacted at: tntam@ctu.edu.vn

For instructions on how to order reprints of this article, please visit our website: www.emeraldgrouppublishing.com/licensing/reprints.htm

Or contact us for further details: permissions@emeraldinsight.com 